\title{
HIV Infection among Newly Diagnosed TB Patients in Southwestern Nigeria: A Multi-DOTS Center Study*
}

\author{
Georgina N. Odaibo $^{1 \#}$, Prosper Okonkwo ${ }^{2}$, Oluwole M. Lawal ${ }^{3}$, David O. Olaleye ${ }^{1}$ \\ ${ }^{1}$ Department of Virology, College of Medicine, University of Ibadan, Ibadan, Nigeria; ${ }^{2}$ AIDS Prevention Initiative Nigeria, Abuja, \\ Nigeria; ${ }^{3} \mathrm{~TB}$ and Leprosy Control Unit, Oyo State Ministry of Health, Ibadan, Nigeria.

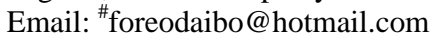

Received January $20^{\text {th }}, 2013$; revised February $21^{\text {st }}, 2013$; accepted February $28^{\text {th }}, 2013$

Copyright (C) 2013 Georgina N. Odaibo et al. This is an open access article distributed under the Creative Commons Attribution License, which permits unrestricted use, distribution, and reproduction in any medium, provided the original work is properly cited.

\begin{abstract}
Backgroud: The burden of TB and HIV infection is estimated to be about 512/100,000 and 3,000,000 people respectively. However, accurate data on TB/HIV co-morbidity in different parts of Nigeria were not available due to limited access to HIV Counseling and Testing (HCT) by individuals with TB infection. This study was designed to determine the true rate of HIV infection among newly diagnosed TB patients by providing comprehensive HCT services in 43 DOTS centers in Oyo State, Southwestern Nigeria. Methods: All patients meeting the case definition for TB suspects who presented at each of the 43 DOTS Centres were counselled and those who consented tested for presence of HIV antibodies using HIV 1/2 Determine ${ }^{\circledR}$, Unigold ${ }^{\circledR}$ and Stat-Pak ${ }^{\circledR}$ rapid test kits in a serial algorithm. Results: A total of 13,109 TB positive patients were enrolled for the study, out of which 1605 (12.3\%) tested positive for HIV antibodies. HIV infection was higher among female (15.5\%) than male (9.5\%) TB patients $(\mathrm{p}<0.05)$. The rates also varied among the age groups, ranging from $4.3 \%$ in the $10-19$ years to $18.0 \%$ in the $40-49$ years age group. A relatively high rate (10.5\%) of HIV infection was found among children less than ten years of age. Conclusion: The results of this work show the true burden of TB/HIV in any region in Nigeria for the first time. Higher rate of TB/HIV co-infection among female patients and children are significant and important factors that should be considered in the planning of intervention measures in Nigeria and other TB and HIV endemic countries in Africa.
\end{abstract}

Keywords: HIV; TB; Co-Infection; DOTS Centre Attendees; Nigeria

\section{Introduction}

Recent studies have indicated resurgence of TB infection after many decades of decline in its incidence. This resurgence has been attributed to the high rate of HIV in-

\footnotetext{
*Ethical considerations: Approval for the study was obtained from the Oyo state ministry of health ethical review board. Informed consent was obtained from all participants in the study.

Authors' contribution: GNO and DOO conceived the idea of the study, GNO, PO, OML and DOO were involved in collection and analysis of data, PO supervised the M \& E aspect of the work; OML recruited and enrolled the patients, GNO and DOO supervised the HIV and TB testing. GNO wrote the draft manuscript while all authors reviewed the final manuscript and approved the final version. DOO is the guarantor of the paper.

Funding: The study was carried out with funding from AIDS Prevention Initiative Nigeria (APIN)/PEPFAR as part of a grant on HCT in DOTS Centres in Oyo state (Oyo 43 DOTS Project).

Competing interest: We declare that we have no conflicting interest in the conduct of the study.

Data sharing: Complete data from this study is available to the funder, Oyo State Ministry of Health and Federal Ministry of Health of Nigeria.

\#Corresponding author.
}

fection in countries with high TB burden. Globally, the rate of new TB infection is about $9 \%$ but it is as high as $31 \%$ in regions and countries with high rate of HIV infection [1]. While the prevalence of HIV infection among TB patients also varies from country to country, the highest rates have been reported in areas where HIV prevalence is high in the general population. For example a high co-infection rate of $44 \%-52 \%$ has been reported in Kenya [2], while a lower co-infection rate of $8.8 \%$ was obtained in Southern California, USA.

Since the first case of AIDS was reported in Nigeria in 1986, the rate of HIV infection among pregnant women attending ante natal clinics steadily increased from $0.6 \%$ in 1987 to $5.8 \%$ in 2003. Although, the 2010 National sentinel survey showed that the rate of HIV infection has declined to $3.4 \%$ [3], the prevalence varies by location from $2.1 \%$ and $2.9 \%$ in the north central and south western zone respectively to $7.5 \%$ in the north central zone. The rate of the infection is higher among some other 
groups/population, especially the high risk groups [4,5]. The year 2000 national survey among TB patient showed a median HIV prevalence rate of $17.0 \%$ with the highest and lowest rates of $35.1 \%$ and $4.2 \%$ in Benue state and Oyo state respectively.

The effect of HIV-TB co-infection is bidirectional and synergistic/aggravating [1]. HIV infection compromises the immune system which in turn increases the risk of acquiring TB. On the other hand, the course of HIV infection is often accelerated subsequent to infection with TB [6]. Hence, the risk of death and development of other opportunistic infections is higher in HIV-TB coinfection and high case fatality rates are observed among tuberculosis patients with HIV infection $[7,8]$.

The WHO guideline of 2004 [9] recommends that all countries with a generalized HIV epidemic (HIV prevalence $>5 \%$ ) should aim to ensure that HIV counseling and testing (HCT) are actively promoted and offered to all TB patients [9]. This recommendation has not been fully implemented in Nigeria due to some technical and logistic challenges such as dearth of trained health workers to carry out HIV testing in all the TB diagnostic and treatment centres, availability of test kits, and other consumables required for HCT. Most TB patients in the country are referred to HCT centres, mainly in secondary and tertiary hospitals where reagents and consumables for HIV testing are regularly available but with the consequence of many of the patients being lost to follow-up. This study was therefore designed to determine the rate of HIV-TB co-in- fection using point of care HCT strategy for newly diagnosed TB cases among patients attending 43 DOTS centres in some urban and rural settings in the 33 Local Government Areas (LGA) in Oyo state, southwestern, Nigeria.

\section{Materials and Method}

\subsection{Study Area and Population}

Participants enrolled in this study included patients that met the case definition for TB suspects who consented to participate in the study and provided sputum for TB test in 43 out of the 56 DOTS Centres in Oyo State. Only data from patients whose sputum tested positive for TB were analyzed for this report. The DOTS Centres are located in all 33 LGAs of the State (Figure 1).

\subsection{Inclusion and Exclusion Criteria}

All TB suspects who provided sputum for AFB test were included while those who were unable to produce sputum were excluded from the study.

\subsection{Methodology}

Health workers providing care at the 43 DOTS Centre in Oyo state were trained to provide comprehensive point of care HCT to patients accessing care for TB in the clinics. All patients who attended the clinics from August 2008 to July 2011 were tested for HIV infection using Determine $^{\circledR}$, Unigold $^{\circledR}$ and Stat-pack ${ }^{\circledR}$ rapid test kits in a serial

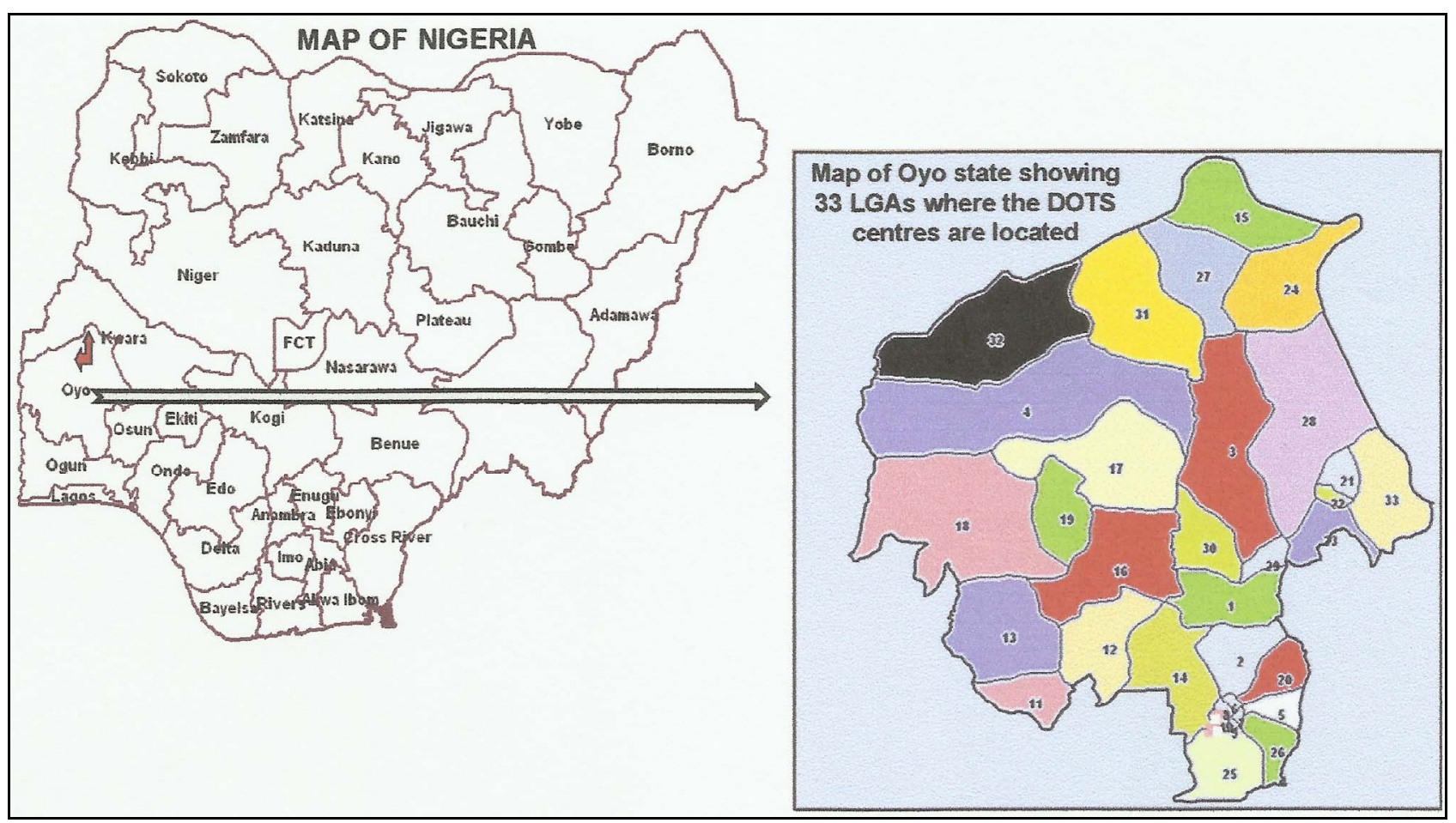

Figure 1. Maps of Nigeria and Oyo State showing location of DOTS centers. 
algorithm. However, only data from those who met the inclusion criteria were analysed. Blood samples were collected by finger pricking and tested for HIV antibodies using the Determine HIV 1/2 rapid test kits. Samples that were reactive by Determine were further tested by Unigold and those with discordant results from the two assays were retested using Stat-pack. All the test procedures were carried out according to the manufacturers' recommendations. Individuals who were reactive in two of the assays were considered as positive for HIV antibodies and were referred to ART Centre of their choice from a list of centres in the state for HIV care and management while those who were positive by Determine ${ }^{\circledR}$ only were requested to represent for re-evaluation in 6 to 12 weeks.

\section{Results}

A total of 13,059 new TB cases were identified in the 43 DOTS Centre during the 3 years period out of which 1617 were HIV positive giving an overall prevalence of HIV infection among the newly diagnosed TB patients of $12.3 \%$. There was no significant difference in the number of new TB cases seen over the years (Table 1). Similar rates of HIV infection was obtained in the first (13.2\%) and second (13.1\%) years but declined significantly to $10.8 \%$ ( $p<0.05$ ) in the third year of the study (Table 1 ). Figure 2 shows the prevalence of HIV infection by the various months over the 3 years. The rates were consistently between $10 \%$ and $15 \%$ with exception of the first two months (August and September 2008) and the months of December and January of the third year during which the highest rates (23.9 and $26.0 \%$ respectively) and lowest rates $(7.7 \%$ and $5.1 \%)$ were obtained respectively.

Figure 3 shows the distribution of HIV/TB co-infection in the different age groups. Overall, the rate of coinfection increased with age from $4.3 \%$ in the $10-19$ years group and peaked at the 30 - 49 years group (16.9\% $18 \%)$. A relatively high rate of HIV infection (10.5\%) was observed among children less than 10 years of age. In addition, the rate was highest among the 30 - 39 years age group in the first and third years and highest among the 40 - 49 years during the second year of the study while the rate of co-infection was consistently lowest among the 15 - 19 years age group.

Although the number of male patients with TB was consistently higher than the female patients, the rate of HIV/TB co-infection was consistently higher among female over the 3 years period (Table 1). Overall, the prevalence

Table 1. Distribution of HIV Infection by Gender among TB Patients in Oyo State, Nigeria.

\begin{tabular}{|c|c|c|c|c|c|c|c|c|}
\hline \multirow{2}{*}{ Female } & \multicolumn{2}{|c|}{ Year One } & \multicolumn{2}{|c|}{ Year Two } & \multicolumn{2}{|c|}{ Year Three } & \multicolumn{2}{|c|}{ Overall } \\
\hline & No Tested & No (\%) Positive & No Tested & No (\%) Positive & No Tested & No (\%) Positive & No Tested & No (\%) Positive \\
\hline Female & 1981 & 304 (15.3) & 2045 & 339 (16.6) & 1988 & $290(14.6)$ & 6014 & 933 (15.5) \\
\hline Total & 4148 & 549 (13.2) & 4477 & 585 (13.1) & 4484 & 483 (10.8) & 13109 & 1605 (12.2) \\
\hline
\end{tabular}

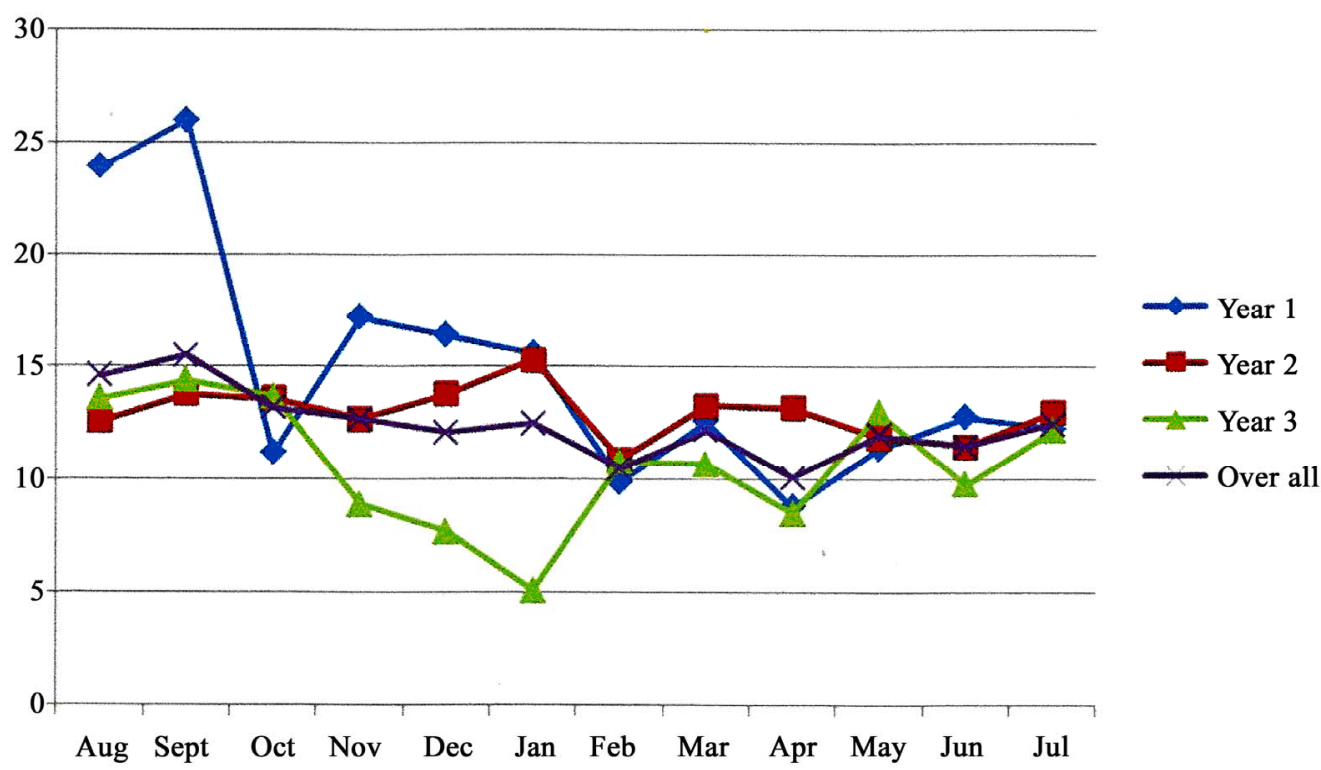

Figure 2. Monthly distribution of HIV infection among TB patients in Oyo State, Nigeria. 


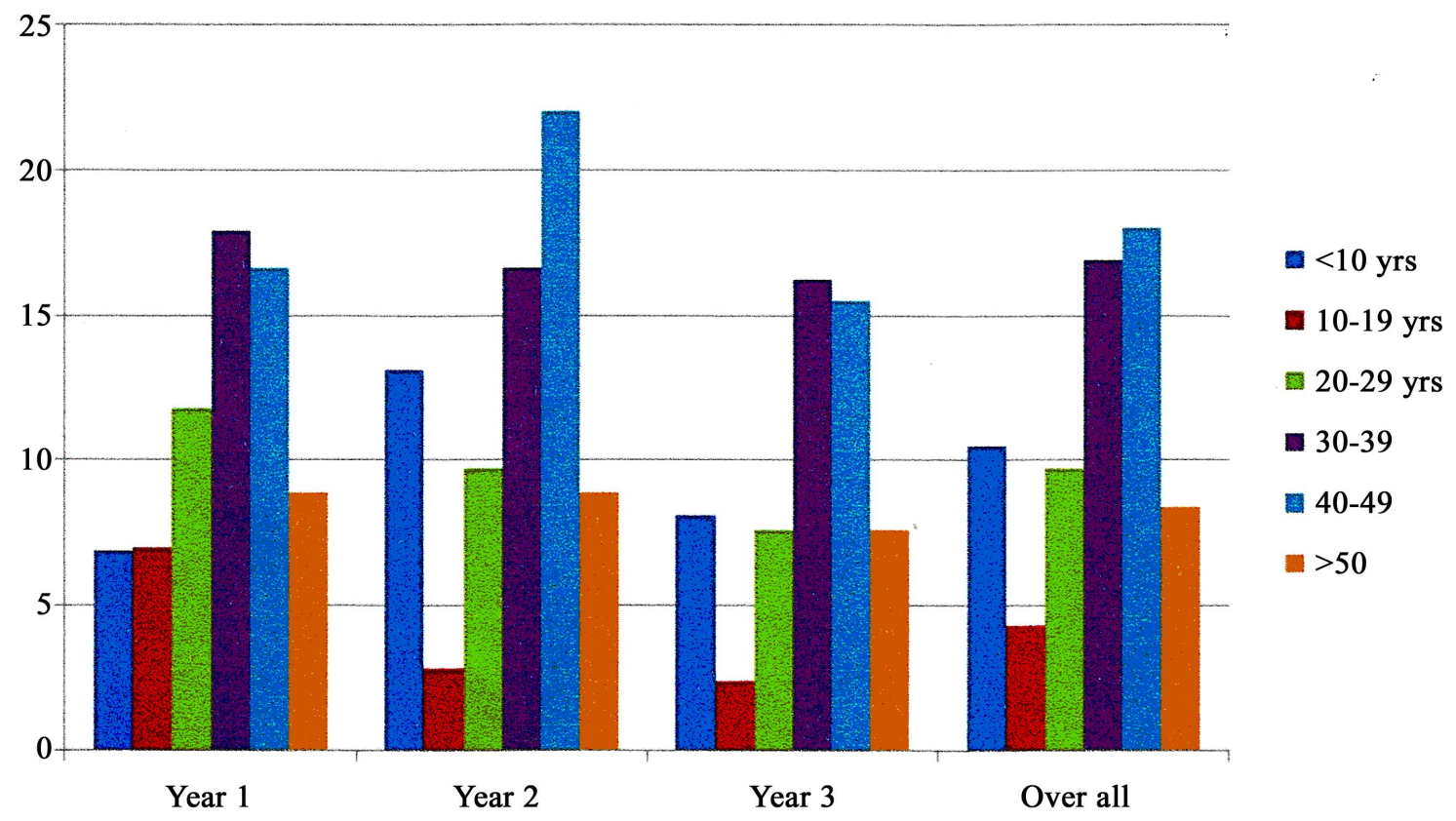

Figure 3. HIV infection by age among newly diagnosed TB patients in Oyo State.

was $9.5 \%$ and $15.5 \%$ for male and female respectively (p $<0.05$ ) giving a male to female ratio of 1:1.6.

\section{Discussion}

The results of this work show a high burden of HIV/TB co-infection in Oyo state, southwestern Nigeria and the first report of a community based HIV/TB burden in the country. The overall prevalence of HIV infection among newly diagnosed TB patients of $12.3 \%$ is higher than previously reported among this category of patients in the state [10]. The national survey of HIV infection among PTB patients conducted in 2000 showed that the rate of HIV/TB co-infection was $4.2 \%$ in Oyo state. Although samples used in that national sentinel survey was collected from only two health facilities and within 8 weeks unlike the current study that was carried out over a period of 3 years and from 43 health facilities and state wide coverage. This increased rate of HIV/TB co-infection in Oyo state may be due to an increase in the rate of HIV infection from $0.1 \%$ in 1991 to 3.9 in the 2003 and 2.2 in 2008 among the general population [4,11]. HIV infection is known to compromise the immune systemthereby increasing the rate of acquiring tuberculosis [10, 12].

The rate of $12.3 \%$ found in this study is similar to recent report from Sagamu and Ile-Ife, also in southwestern Nigeria but differs greatly from the rates found in studies from other regions of the country with higher HIV prevalence rates in the general population. For example Pennap et al. [13] and Erhabor et al. [14] reported a co-infection rate of $44.2 \%$ and $25 \%$ from Nasarawa in the north central and Port Harcourt in the south-south region of the country respectively. This difference further confirms the correlation between HIV infection rates in the general population and the development of TB following exposure [10], i.e. the higher the rate of HIV infection, the greater the risk of acquiring established TB infection and hence a higher rate of HIV/TB co-infection, especially in areas with high TB endemicity.

Although the number of newly diagnosed TB cases was similar in the 3 years of the study, a reduction in the co-infection rate was observed in the 3rd year of the study. This decline which is in consonant with the results of the 2008 and 2010 national survey [3,11] may be a reflection of increased awareness and practice of prevention strategies at the community level in the state. However, continuation of the study for additional one to two years is necessary to confirm if this reduction in HIV/TB co-infection rate is real.

More males than females were newly diagnosed with TB in the communities where this study was carried out. However, the rate of HIV infection was higher among the female TB patients (male to female ratio-1:1.6). This observation is different from what was reported in previous National HIV prevalence surveys where HIV/TB Co-infection male to female ratio of $1: 1$ was obtained [10, 15]. It is however similar to reports of recent studies conducted in other parts of Nigeria [13,14]. Similarly, Sawent et al. [16] and Potororing et al. [8] reported a higher rate of HIV infection in female TB patients than males in India and Indonesia respectively. This shift is an indication that women are now more at risk of acquiring HIV 
infection than men in Nigeria. This rising trend in female HIV prevalence is not unexpected due to the fact that the penile-vaginal transmission by an infected individual in a single sexual exposure is as low as one in 1000 from woman to man and as high as one in 300 from man to woman [17]. In addition, the poor economic status and the pressure on women to provide for their families as well as the lack of ability to negotiate safer sex by Nigeria women [18] may have contributed to increasing the risk of women to acquiring HIV infection and hence the female preponderance.

In this study, HIV prevalence was highest among the age group 30 - 49 years in this study. While this finding is consisted with the previous reports from the country as well as other TB endemic countries $[10,13,16,19]$ the pattern is different from the pattern of HIV infection in the general population with the highest infection rate among individuals in the 20 - 29 years age group [3]. This difference in the most affected age group further reflects the role of HIV infection as an important factor in the acquisition of TB in Nigeria. Individuals in this age group make up a greater proportion of the workforce in the country thus the impact of HIV/TB co-infection on the economy of the country will certainly become overwhelming if not controlled with appropriate intervention measures such as early detection and treatment of both infection.

The consistently low HIV infection rate observed in the 10 - 19 age group over the years is in line with previous national data [10] and may indicate less HIV associated acquisition of TB in this age group. On the other hand, the high rate of HIV infection among TB patient below 10 years of age is worrisome. As previously observed [10], this may be due to uncontrolled high rate of mother to child transmission of HIV in the country, with concurrent horizontal transmission of TB from adults to children in a household and communities. Although prevention of mother to child transmission (PMTCT) program has been in operation in Nigeria since 2002, it is still not universally accessible especially in semi-urban and rural communities. The challenge of accessibility of HIV care coupled with poor health seeking behavior leading to several out of hospital births may have contributed to the increase of HIV \& TB co-infection among this very young age group.

We conclude that the rate of HIV infection among patients newly diagnosed with TB in Oyo State, Nigeria is remarkably higher than previously reported. In addition, consistent with reports from some other communities, the rate of TB/HIV co-infection among female patients and children was remarkably high. These are important factors that should be considered in the planning of intervention measures in the country as well as in other countries where both infections are endemic.
What is already known on this subject?

- HIV/TB co-infection rate is on the increase especially in countries where both infections are endemic.

- WHO recommends that HIV counseling and testing be offered to all TB patients in countries with generalized HIV epidemic such as Nigeria.

- Accessibility to HIV Counseling and testing (HCT) by patients with TB is a major hindrance to achieving the above WHO recommendation in sub Saharan Africa.

- Most studies on HIV/TB in Nigeria have been done in secondary and tertiary hospital settings.

What this study adds to literature?

- The true burden of HIV/TB co-infection based on data collected from 43 DOTS centres located within communities in Nigeria is reported for the first time.

- The study shows a remarkably high rate of HIV infection among patients newly diagnosed with TB in Nigeria.

- High rate of HIV/TB co-infection among female patients and children are significant and therefore important factors that should be considered in the planning of intervention measures in Nigeria and other countries where TB and HIV are endemic.

\section{Acknowledgements}

This publication was supported by Cooperative Agreement No: 1U2GPS001058 from the Centers for Disease Control and Prevention. Its content are solely the responsibility of the authors and do not necessarily represent the official views of the Centers for Disease Control and Prevention.

We express sincere gratitude to all the staff of the Oyo 43 DOTS Project, the TBL supervisors for patient enrollment and carrying out HIV testing. We are also very grateful to the patients who participated in the study.

\section{REFERENCES}

[1] S. K. Sharma, A. Mohan and T. Kadhiravan, "HIV-TB CoInfection: Epidemiology, Diagnosis and Management," Indian Journal of Medical Research, Vol. 121, No. 4, 2005, pp. 550-567.

[2] Center for Disease Control and prevention (CDC), "HIV Testing and Treatment among Tuberculosis Patients-Kenya 2006-2009,” JAMA, Vol. 305, No. 4, 2011, pp. 351-353.

[3] FMOH, "National HIV Sero-Prevalence Sentinel Survey among Pregnant Women Attending Antenatal Clinics in Nigeria,” Technical Report, 2010, pp. 1-96.

[4] FMOH, “A Technical Report on 2001 HIV/Syphilis Sentinel Survey among Pregnant Women Attending Antenatal Clinics in Nigeria,” Federal Ministry of Health, Na- 
tional AIDS Control Programmes, 2001, pp. 1-63.

[5] O. M. Ige, O. M. Sogaolu and O. A. Ogunlade, "Pattern of Presentation of Tuberculosis and the Hospital Prevalence of Tuberculosis and HIV Co-Infection in University College Hospital, Ibadan: A Review of Five Years (19982002)," African Journal of Medicine and Medical Sciences, Vol. 34, No. 4, 2005, pp. 329-333.

[6] A. Mahajan and V. R. Tandon, "HIV/AIDS-TB Co-Infection: What Prevalence Indicates?” JK Science Journal of Medical Education and Research, Vol. 9, No. 2, 2007, pp. 56-57.

[7] C. T. Kang'ombe, A. D. Harries, K. Ito, et al., "Longterm Outcome in Patients Registered with Tuberculosis in Zomba, Malawi: Mortality at 7 Years According to Initial HIV Status and Type of TB," International Journal of Tuberculosis and Lung Disease, Vol. 8, No. 7, 2004, pp. 829-836.

[8] J. G. Pontororing, E. Kenangalem, D. B. Lolong, G. Waramori, Sandjaja, E. Tjitra, R. N. Price, P. M. Kelly, N. M. Anstey and A. P. Ralph, "The Burden and Treatment of HIV in Tuberculosis Patients in Papua Province, Indonesia: A Prospective Observational Study,” BioMed Central Infectious Diseases, Vol. 10, 2010, p. 362. http://www.biomedcentral.com/1471-2334/10/362

[9] World Health Organization, "Interim Policy on Collaborative TB/HIV Activities,” World Health Organization, Geneva, 2004.

http://whqlibdoc.who.int/hq/2004/who_htm_tb_2004.330_ eng.pdf

[10] G. N. Odaibo, M. F. Gboun, E. E. Ekanem, S. N. Gwarzo, I. Saliu, S. A. Egbewunmi, E. A. Abebe and D. O. Olaleye, "HIV Infection among Patients with PTB in Nigeria," African Journal of Medicine and Medical Sciences, Vol. 35, 2006, pp. 93-98.

[11] FMOH, "Sero-Prevalence Sentinel Survey among Pregnant Women Attending Antenatal Clinics in Nigeria,” A Technical Report, Federal Ministry of Health, National
AIDS Control Programmes, 2008, pp. 1-85.

[12] G. Pennap, S. Makpa and S. Ogbu, "Sero-Prevalence of HIV Infection among Tuberculosis Patients in a Rural Tuberculosis Referral Clinic in Northern Nigeria,” Pan African Medical Journal, Vol. 5, 2010, p. 22.

[13] O. Erhabor, Z. A. Jeremiah, A. C. Adias and C. E. Okere, "The Prevalence of Human Immunodeficiency Virus Infection among TB Patients in Port Harcourt Nigeria," HIV/ AIDS-Research and Palliative Care, Vol. 2, 2010, pp. 1-5.

[14] FMOH, "HIV Sero-Prevalence Sentinel Survey among Pregnant Women Attending Antenatal Clinics in Nigeria,” A Technical Report, Federal Ministry of Health, National AIDS Control Programmes, 2003, pp. 1-63.

[15] S. S. Sawant, S. R. Agrawal, J. S. Shastri, M. Pawaskar and P. Kadam, "Human Immunodeficiency Virus Infection among Tuberculosis Patients in Mumbai," Journal of Laboratory Physicians, Vol. 3, No. 1, 2011, pp. 12-14.

[16] J. C. Cadwell, "Understanding the AIDS Epidemic and Reacting Sensibly to It," Social Sciences and Medicine, Vol. 41, No. 3, 1995, pp. 299-302.

[17] C. O. Isiramen, "Women in Nigeria: Religion, Culture and AIDS,” International Humanist News and Ethical Union, 2003.

http://iheu.org/content/women-nigeria-religion-culture-an d-aids

[18] Z. Iliyasu and M. Babashani, "Prevalence and Predictors of TB Coinfection among HIV Seropositive Patients Attending Aminu Kano Teaching Hospital, Northern Nigeria,” Journal of Epidemiology \& Community Health, Vol. 19, No. 2, 2009, pp. 81-87.

[19] T. C. Rodwell, R. F. W. Barnes, M. Moore, S. A. Strathdee, A. Raich, K. S. Moser and R. S. Garfein, "HIV-Tuberculosis Coinfection in Southern California: Evaluating Disparities in Disease Burden," American Journal of Public-Health, Vol. 100, Suppl. 1, 2010, pp. S178-S185. 